\title{
Association of LPP and TAGAP Polymorphisms with Celiac Disease Risk: A Meta-Analysis
}

\author{
Shi-Qi Huang ${ }^{1,+}{ }^{\dagger}$ Na Zhang ${ }^{1,2,+}{ }^{\dagger}$ Zi-Xing Zhou ${ }^{1}$, Chui-Can Huang ${ }^{1}$, Cheng-Li Zeng ${ }^{1}$, Di Xiao ${ }^{1}$, \\ Cong-Cong Guo ${ }^{1}$, Ya-Jing Han ${ }^{1}$, Xiao-Hong Ye ${ }^{1}$, Xing-Guang Ye ${ }^{1}$, Mei-Ling Ou ${ }^{1}$, \\ Bao-Huan Zhang ${ }^{1}$, Yang Liu ${ }^{1}$, Eddy Y. Zeng ${ }^{3}$, Guang Yang ${ }^{3,4, *}$ and Chun-Xia Jing ${ }^{1,3, *}$ \\ 1 Department of Epidemiology, School of Basic Medical Sciences, Jinan University, \\ No. 601 Huangpu Road West, Guangzhou 510632, Guangdong, China; cookiehaha@sina.cn (S.-Q.H.); \\ lilyronna@outlook.com (N.Z.); m13682215478@163.com (Z.-X.Z.); huangcc531@163.com (C.-C.H.); \\ chengleezeng@163.com (C.-L.Z.); kwstljh@163.com (D.X.); guo301@aliyun.com (C.-C.G.); \\ yajinghan001@outlook.com (Y.-J.H.); 15521143958@163.com (X.-H.Y.); yestar1989@163.com (X.-G.Y.); \\ oumeiling1214@outlook.com (M.-L.O.); candy2006520@163.com (B.-H.Z.); liuyang199101@126.com (Y.L.) \\ 2 Department of Preventive Medicine, Zunyi Medical College, Zhuhai Campus, Zhuhai 519041, \\ Guangdong, China \\ 3 School of Environment, Guangzhou Key Laboratory of Environmental Exposure and Health, \\ Guangdong Key Laboratory of Environmental Pollution and Health, Jinan University, \\ Guangzhou 510632, Guangdong, China; eddyzeng@jnu.edu.cn \\ 4 Department of Parasitology, School of Basic Medical Sciences, Jinan University, \\ Guangzhou 510632, Guangdong, China \\ * Correspondence: guangyangphd@gmail.com (G.Y.); tjchx@jnu.edu.cn (C.-X.J.); \\ Tel.: +86-20-8522-0255 (G.Y.); +86-20-8522-0258 (C.-X.J.); Fax: +86-20-8522-1343 (G.Y. \& C.-X.J.) \\ + These authors contributed equally to this work.
}

Academic Editor: Paul B. Tchounwou

Received: 1 December 2016; Accepted: 31 January 2017; Published: 10 February 2017

\begin{abstract}
Background: Lipoma preferred partner (LPP) and T-cell activation Rho GTPase activating protein (TAGAP) polymorphisms might influence the susceptibility to celiac disease. Therefore, we performed a meta-analysis by identifying relevant studies to estimate the risks of these polymorphisms on celiac disease. Methods: The PubMed, Web of Science and Embase databases were searched (up to October 2016) for LPP rs1464510 and TAGAP rs1738074 polymorphisms. Results: This meta-analysis included the same 7 studies for LPP rs1464510 and TAGAP rs1738074. The minor risk A allele at both rs1464510 and rs1738074 carried risks (odds ratios) of 1.26 (95\% CI: 1.22-1.30) and 1.17 (95\% CI: 1.14-1.21), respectively, which contributed to increased risks in all celiac disease patients by $10.72 \%$ and $6.59 \%$, respectively. The estimated lambdas were 0.512 and 0.496 , respectively, suggesting that a co-dominant model would be suitable for both gene effects. Conclusions: This meta-analysis provides robust estimates that polymorphisms in LPP and TAGAP genes are potential risk factors for celiac disease in European and American. Prospective studies and more genome-wide association studies (GWAS) are needed to confirm these findings, and some corresponding molecular biology experiments should be carried out to clarify the pathogenic mechanisms of celiac disease.
\end{abstract}

Keywords: LPP; TAGAP; polymorphism; celiac disease; meta-analysis

\section{Introduction}

Celiac disease (CD) is a chronic and immune-mediated enteropathy that is induced by dietary protein gluten (from wheat, barley and rye) in genetically predisposed individuals [1]. It is a small-intestine disorder, affecting approximately $1 \%$ of the European population with some regional 
variations [2] and causing malnutrition and severe complications. Celiac patients have a greater burden of disease than the general population, and a long-term gluten-free diet (GFD) is the only therapy for this disease [1,3]. HLA-DQ2 and HLA-DQ8 molecules are responsible for only approximately $40 \%$ of genetic predisposing factors in the pathogenesis of $\mathrm{CD}$ [4], which is necessary but not sufficient to cause disease $[5,6]$. Thus, many more risk loci outside the HLA region should be identified as disease markers.

In recent years, genome-wide association studies (GWAS) have expanded our understanding of genetic makeup and revealed several possible inherited risk factors in celiac disorders [7-10]. Many of the non-HLA loci overlap with Crohn's disease, type 1 diabetes, rheumatoid arthritis and juvenile idiopathic arthritis [11-15], such as lipoma preferred partner $(L P P)$ and T-cell activation Rho GTPase activating protein (TAGAP). Alterations of the actin cytoskeleton and cell shape can be observed in the CD patients' intestinal mucosa [16,17], while the cell shape is maintained through the actin cytoskeleton and focal adhesion [18]. LPP is localized with paxillin in focal adhesions, and the number of paxillin focal adhesions with $L P P$ is increased in CD fibroblasts. A constitutive alteration in cell shape and adhesion involving $L P P$ occurs in CD fibroblasts, suggesting a correlation between LPP and CD pathogenesis [19]. In addition, $L P P$ is considered a substrate of the protein-tyrosine-phosphatase $1 \mathrm{~B}$ (PTP1B) [20]. Of note, loss of PTP1B can attenuate the activation of extracellular signal regulated kinase (ERK) [21], which is activated in the CD patients' mucosa on a GFD or a gluten-containing diet (GCD). Only when ERK is phosphorylated can it transduce to the nuclei, and it has been found that more nuclei of the enterocytes from CD patients were positive for ERK compared with controls. Inhibition of ERK phosphorylation normalizes crypt enterocyte proliferation of CD atrophic mucosa [22]. When PTP1B is sufficient or excessive, there may be more ERK activity in the celiac enterocytes, resulting in the progression of $\mathrm{CD}$.

TAGAP is involved in the Rho GTPase cycle [23,24], which is between the inactive GDP-bound and the active GTP-bound states. The exchange of GDP-bound for GTP-bound is catalyzed by GEFs, while GAPs increase the intrinsic GTPase activity of Rho GTPases to accelerate the return of the proteins to the inactive state [25-27]. In the active state, GEF-catalyzed activation of Rho interacts with ROCK, which can activate the myosin light chain (MLC) and LIM domain kinase (LIMK), and both of them play an important role in focal adhesion and regulate the rearrangement and stabilization of the actin cytoskeleton [28]. However, TAGAP propagates the inactive form of the RHO molecule; and it increases the activity of Rho GTPases via phosphorylation, enhancing their intrinsic activity up to fivefold [29]. TAGAP negatively regulates downstream effects; thus, the actin cytoskeleton rearrangement is dysfunctional and lack of unstable [23].

Mutation of LPP and TAGAP may interfere with their original function and even promote the progress of $C D$. In recent years, a number of studies, including GWAS, have reported the association of LPP and TAGAP polymorphisms with CD susceptibility, and many have focused on LPP rs1464510 (A/C) and TAGAP rs1738074 (A/G). However, those studies have drawn inconsistent conclusions due to the limited regions and small numbers of articles. For example, Dubois et al. [8] reported that rs1464510 was positively associated with CD in the Netherlands, whereas there was no relationship in a Dutch population according to Coenen et al. [30] and Hunt et al. [9]. Similarly, results for rs1738074 differed from country to country in the studies by Plaza-Izurieta et al. [7] and Sperandeo et al. [31]. Therefore, we decided to carry out this meta-analysis on all the available case-control studies to accurately assess the relationship between the LPP rs1464510/TAGAP rs1738074 and CD risk.

\section{Materials and Methods}

\subsection{Search Strategy}

Relevant studies were searched in PubMed, the Web of Science and Embase up to October 2016. The search strategies were as followed: (( $L P P$ or 3q28 or rs1464510 or "lipoma preferred partner") or "lim domain containing preferred translocation protein") and celiac disease) or ((TAGAP or 6q25 
or rs1738074 or "T-cell activation GTPase activating protein") and celiac disease). The search was limited to English-language and human studies. Only published studies were considered. We scanned the title and abstract of all relevant articles, manually examined reference lists for additional relevant publications and obtained the full text of all possibly relevant studies. If multiple articles were published on the same topic, the most complete and recent study was used.

\subsection{Inclusion and Exclusion Criteria}

A reviewer independently examined the titles and abstracts of the identified articles. Any human population-based association study was included regardless of subjects' ethnicity if it met the following criteria: (1) it showed an association between LPP (rs1464510) or TAGAP (rs1738074) polymorphism, (2) the outcome was celiac disease and there was a control group, (3) there were sufficient data for extraction (i.e., minor allele frequency and genotype frequency) and (4) there was a clear diagnosis of celiac disease. Studies were excluded if: (1) the case and control subjects were biologically related; (2) the insufficient data that were failed to ask for supplementary information from the authors; (3) the studies comprised unrelated data, family studies, animal studies, reviews, or meeting abstracts; or (4) the studies were written not in English.

\subsection{Data Extraction}

Summary data were extracted independently by reviewers using a standardized data extraction form. We extracted general information as follows: name of first author, year of publication, region of study population, source of controls, genotype method, diagnostic criteria, the number of cases and controls, and the minor allele frequency in cases and controls. Any disagreement was resolved by consensus.

\subsection{Risk of Bias Assessment}

Study quality was assessed independently by the same reviewers using a risk-of-bias score for genetic association studies that was developed by Thakkinstian et al. [32] (Supplementary Materials Table S1). The score considered 5 domains: information bias (ascertainment of outcome and gene), confounding bias, selective reporting of outcomes, population stratification, and Hardy-Weinberg equilibrium (HWE) assessment in the control group. Each item was scored "yes", "no" or "unclear", representing low risk, high risk and insufficient information, respectively. Disagreement between the two reviewers was solved by a senior reviewer (C.X.J). Additionally, the MOOSE checklist was used to measure the quality of our study (Supplementary Materials Table S2).

\subsection{Statistical Analysis}

We used Stata software (version 12.0, StataCorp LLC, College Statopm, TX, USA) and the Comprehensive Meta-Analysis software (version 2.0, Biostat, Englewood, NJ, USA) for all statistical analyses. All tests with a $p$ value less than 0.05 were considered statistically significant, except for the heterogeneity tests, in which a $p$ value less than 0.10 was used. It was tested whether the distribution of genotypes in the controls was compliant with Hardy-Weinberg equilibrium (HWE) by a Fisher's exact test to estimate the quality of studies. If the study was found not to be in HWE with a $p$ value less than 0.05 , it was considered to be in disequilibrium. We used both per-allele and per-genotype analysis to estimate the strength of the association between the polymorphism of $L P P$ rs1464510 or TAGAP rs1738074 and CD risks.

Per-allele analysis: Suppose that A and a are risk and non-risk alleles, respectively, and AA, Aa and aa are minor homozygous, heterozygous, and common homozygous genotypes, respectively, for each polymorphism. The risk allele frequency in each group was estimated according to the reported genotype data, and overall prevalence along with $95 \%$ confidence intervals were estimated for each single nucleotide polymorphism (SNP). The Mantel-Haenszel method was used to determine the statistical significance of the pooled OR, and its $p$ value was used to determine whether the overall 
gene effect was significant $(p=0.05)$. The heterogeneity of allele effects across studies was checked using a $Q$ test, and the degree of heterogeneity was quantified by $\mathrm{I}^{2}\left(\mathrm{I}^{2}<25 \%\right.$, no heterogeneity; $25 \%<$ $\mathrm{I}^{2}<50 \%$, moderate heterogeneity; $50 \%<\mathrm{I}^{2}<75 \%$, large heterogeneity; $\mathrm{I}^{2}>75 \%$, extreme heterogeneity). If heterogeneity was present (i.e., if the $Q$ test was significant or $I^{2}$ was greater than $25 \%$ ), the cause of heterogeneity was explored using sensitivity analysis. We chose a random-effects model if $\mathrm{I}^{2}$ was greater than $50 \%$; otherwise, a fixed-effects model was used. The population attributable risk (PAR) for the risk allele was calculated based on results from a discrete-time model. If the main effect of the genotype was statistically significant and had the appropriate effect model selection, further comparisons of $\mathrm{OR}_{1}$ and $\mathrm{OR}_{2}$ were explored. Per-genotype analysis: We used the model-free approach to estimate the genotype effect, and two odds ratios-AA vs. aa $\left(\mathrm{OR}_{1}\right)$ and Aa vs. aa $\left(\mathrm{OR}_{2}\right)$-were estimated for each study. The model of the genetic effect, measured by the parameter lambda $(\lambda)$, which is defined as the ratio of $\log \mathrm{OR}_{2}$ to $\log \mathrm{OR}_{1}$, was then estimated using the model-free Bayesian approach. Lambda $(\lambda)$ represents the heterozygote effect as a proportion of the homozygote variant effect. The value of lambda ranges from 0 to 1 . We obtained information about the genetic mode of action as follows: If $\lambda=0$, a recessive ( $\mathrm{Aa}+$ aa vs. AA) model is suggested; if $\lambda=1$, a dominant model (AA + Aa vs. aa) is suggested; and if $\lambda=0.5$, a co-dominant model (AA vs. aa, Aa vs. aa) is suggested. If $\lambda>1$ or $\lambda<0$, then a homozygous or heterosis model is likely, although this is rare. Once the best genetic model is identified, this model is used to collapse the three genotypes into two groups and to pool the results again. For lambda, WinBugs 1.4.2 was used with vague prior to distributions for the estimation of parameters (i.e., lambda and odds ratio). The models were run with a burn-in of 1000 iterations, followed by 10,000 iterations for parameter estimates. The Begg and Mazuma rank correlation and Egger's test were adopted to assess and quantify the publication bias. A sensitivity analysis was performed, and we removed studies one by one to reflect the influence of each study on the pooled OR of the others. In addition, we calculated the classic fail-safe $\mathrm{N}$ value using Comprehensive Meta-Analysis software (version 2.0) to quantitatively evaluate the reliability of the results.

\section{Results}

\subsection{Identifying Relevant Studies}

Twenty-five, twenty-one and twenty-five studies were identified from PubMed, Web of Science, and Embase, respectively; an additional three studies were identified from references in the included studies (Figure 1). After duplicates were removed, there were forty-eight studies, thirty-nine of which were ineligible. The ineligible records consisted of seventeen other studies, one animal study, three review articles, three family studies, six meeting articles, one meta-analysis of inflammatory bowel disease, two studies without the target SNPs, and six studies aimed at other immune diseases. After retrieving and reviewing the nine remaining studies, we excluded two studies without sufficient data, leaving seven studies to be used for further data extractions (Table 1). 


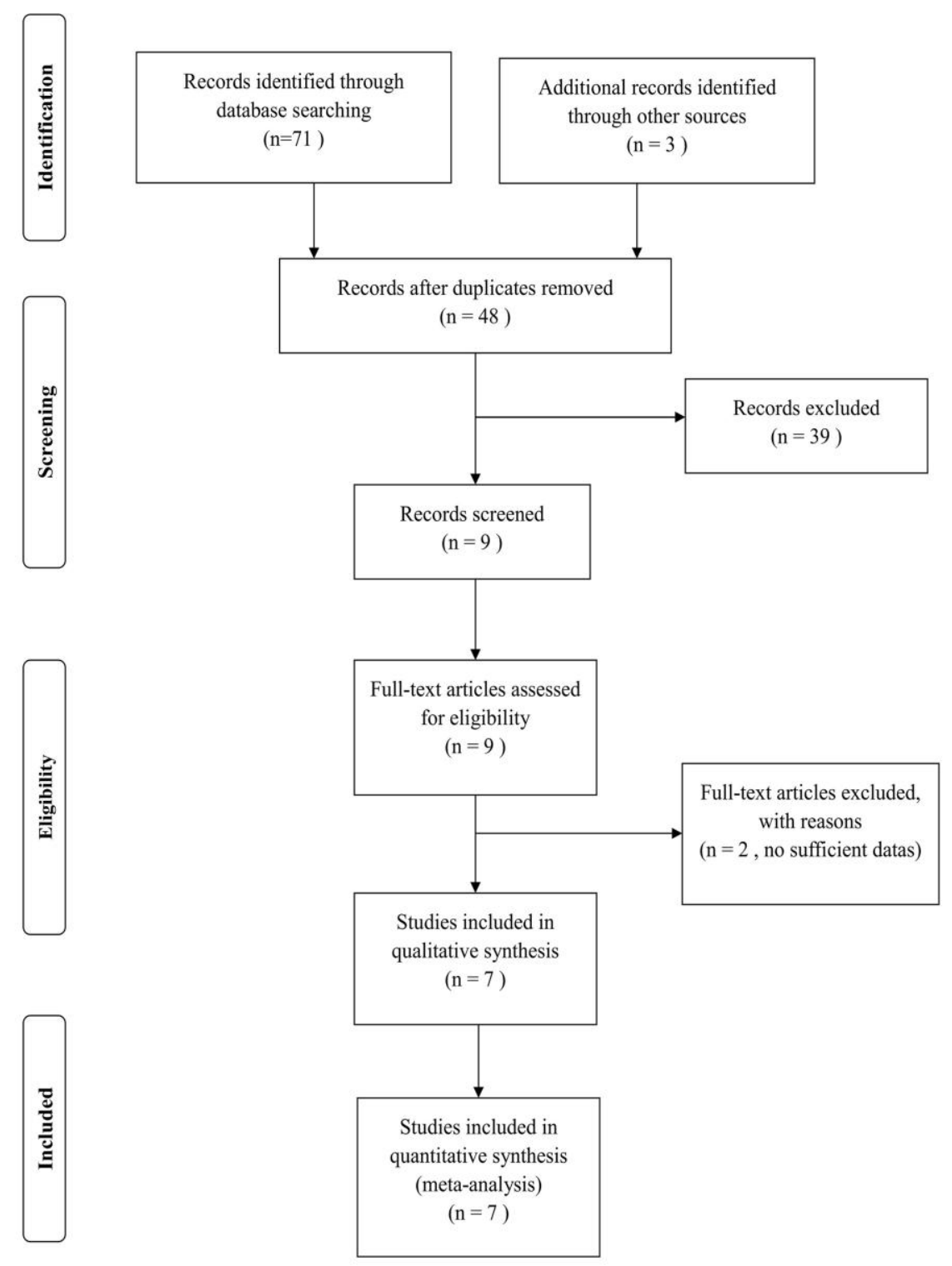

Figure 1. Flow chart for identified studies for $L P P$ and TAGAP genes with CD. 
Table 1. Characteristics of the eligible studies for $L P P$ and TAGAP in meta-analysis.

\begin{tabular}{|c|c|c|c|c|c|c|c|c|}
\hline \multirow{2}{*}{ Authors, Year (Ref.) } & \multirow{2}{*}{ Ethnicity } & \multirow{2}{*}{ Genotype Method } & \multirow{2}{*}{ Gene } & \multirow{2}{*}{ Type of SNP } & \multicolumn{2}{|c|}{ MAF } & \multicolumn{2}{|c|}{ Sample Size } \\
\hline & & & & & Case & Control & Case & Control \\
\hline \multirow{2}{*}{ Plaza-Izurieta et al., 2011 [7] } & \multirow{2}{*}{ Spanish } & \multirow{2}{*}{ RT-PCR } & $L P P$ & rs1464510 & 0.450 & 0.419 & \multirow{2}{*}{1094} & \multirow{2}{*}{540} \\
\hline & & & TAGAP & rs1738074 & 0.423 & 0.406 & & \\
\hline \multirow{2}{*}{ Sperandeo et al., 2011 [31] } & \multirow{2}{*}{ Italian } & \multirow{2}{*}{ TaqMan } & $L P P$ & rs1464510 & 0.493 & 0.406 & \multirow[b]{2}{*}{637} & \multirow{2}{*}{711} \\
\hline & & & TAGAP & rs1738074 & 0.465 & 0.425 & & \\
\hline \multirow{20}{*}{ Dubois et al., 2010 [8] } & \multirow[b]{2}{*}{ British } & Illumina Hap300v1-1 + & $L P P$ & rs1464510 & 0.522 & 0.450 & \multirow{2}{*}{737} & \multirow{2}{*}{2596} \\
\hline & & IlluminaHap550-2v3 & TAGAP & rs1738074 & 0.472 & 0.438 & & \\
\hline & \multirow{2}{*}{ British } & Illumina 670-QuadCustom_v1 + & $L P P$ & rs1464510 & 0.524 & 0.448 & \multirow[b]{2}{*}{1849} & \multirow[b]{2}{*}{4936} \\
\hline & & Illumina 1.2MDuoCustom_v1 & TAGAP & rs1738074 & 0.475 & 0.438 & & \\
\hline & \multirow{2}{*}{ Finnish } & Illumina 670-QuadCustom_v1 + & $L P P$ & rs1464510 & 0.601 & 0.547 & \multirow{2}{*}{647} & \multirow[b]{2}{*}{1829} \\
\hline & & Illumina610-Quad & TAGAP & rs1738074 & 0.430 & 0.421 & & \\
\hline & \multirow{2}{*}{ Dutch } & \multirow{2}{*}{ Illumina 670-QuadCustom_v1 } & $L P P$ & rs1464510 & 0.531 & 0.493 & \multirow{2}{*}{803} & \multirow{2}{*}{846} \\
\hline & & & TAGAP & rs 1738074 & 0.445 & 0.395 & & \\
\hline & \multirow[b]{2}{*}{ Italian } & Tluming 670 Ouad Cuctom 1 & $L P P$ & rs1464510 & 0.517 & 0.472 & & \\
\hline & & Illumina 6/0-QuadCustom_v1 & TAGAP & rs1738074 & 0.464 & 0.413 & 497 & 543 \\
\hline & & & $L P P$ & rs1464510 & 0.511 & 0.459 & & \\
\hline & American & IlluminaGoldenGate & TAGAP & rs1738074 & 0.470 & 0.423 & 973 & 555 \\
\hline & & & $L P P$ & rs1464510 & 0.533 & 0.475 & & \\
\hline & Hungarıan & IlluminaGoldenGate & TAGAP & rs1738074 & 0.415 & 0.372 & 965 & 1067 \\
\hline & & & $L P P$ & rs1464510 & 0.501 & 0.443 & & \\
\hline & Irish & IlluminaGoldenGate & TAGAP & rs1738074 & 0.500 & 0.462 & 597 & 1456 \\
\hline & Polich & Jlumino Coldonfatt & $L P P$ & rs1464510 & 0.495 & 0.452 & 564 & 716 \\
\hline & Polish & IlluminaGoldenGate & TAGAP & rs1738074 & 0.364 & 0.328 & 564 & 716 \\
\hline & Snanich & & $L P P$ & rs1464510 & 0.462 & 0.403 & & \\
\hline & Spanish & IlluminaGoldenGate & TAGAP & rs1738074 & 0.443 & 0.400 & 550 & 433 \\
\hline
\end{tabular}


Table 1. Cont

\begin{tabular}{|c|c|c|c|c|c|c|c|c|}
\hline \multirow{2}{*}{ Authors, Year (Ref.) } & \multirow{2}{*}{ Ethnicity } & \multirow{2}{*}{ Genotype Method } & \multirow{2}{*}{ Gene } & \multirow{2}{*}{ Type of SNP } & \multicolumn{2}{|c|}{ MAF } & \multicolumn{2}{|c|}{ Sample Size } \\
\hline & & & & & Case & Control & Case & Control \\
\hline & Italian & IlluminaGoldenGate & $\begin{array}{l}L P P \\
T A G A P\end{array}$ & $\begin{array}{l}\text { rs1464510 } \\
\text { rs1738074 }\end{array}$ & $\begin{array}{l}0.495 \\
0.461\end{array}$ & $\begin{array}{l}0.408 \\
0.425\end{array}$ & 1010 & 804 \\
\hline & Finnish & $\begin{array}{l}\text { IlluminaGoldenGate + } \\
\text { Illumina610-Quad }\end{array}$ & $\begin{array}{l}L P P \\
T A G A P\end{array}$ & $\begin{array}{l}\text { rs1464510 } \\
\text { rs1738074 }\end{array}$ & $\begin{array}{l}0.602 \\
0.448\end{array}$ & $\begin{array}{l}0.531 \\
0.421\end{array}$ & 259 & 653 \\
\hline Coenen et al., 2009 [30] & Dutch & Illumina HAP550 & $\begin{array}{c}\text { LPP } \\
\text { TAGAP }\end{array}$ & $\begin{array}{l}\text { rs1464510 } \\
\text { rs1738074 }\end{array}$ & $\begin{array}{l}0.530 \\
0.440\end{array}$ & $\begin{array}{l}0.510 \\
0.400\end{array}$ & 795 & 1683 \\
\hline Romanos et al., 2008 [33] & Italian & TaqMan technology & $\begin{array}{l}\text { LPP } \\
\text { TAGAP }\end{array}$ & $\begin{array}{l}\text { rs1464510 } \\
\text { rs1738074 }\end{array}$ & $\begin{array}{l}0.520 \\
0.454\end{array}$ & $\begin{array}{l}0.474 \\
0.412\end{array}$ & 538 & 593 \\
\hline Hunt et al., 2008 [9] & British & IlluminaGoldenGate & $\begin{array}{l}\text { LPP } \\
T A G A P\end{array}$ & $\begin{array}{l}\text { rs1464510 } \\
\text { rs1738074 }\end{array}$ & $\begin{array}{l}0.517 \\
0.460\end{array}$ & $\begin{array}{l}0.446 \\
0.428\end{array}$ & 719 & 1561 \\
\hline & Irish & IlluminaGoldenGate & $\begin{array}{l}\text { LPP } \\
\text { TAGAP }\end{array}$ & $\begin{array}{l}\text { rs1464510 } \\
\text { rs1738074 }\end{array}$ & $\begin{array}{l}0.483 \\
0.519\end{array}$ & $\begin{array}{l}0.448 \\
0.468\end{array}$ & 416 & 957 \\
\hline & Dutch & IlluminaGoldenGate & $\begin{array}{l}\text { LPP } \\
T A G A P\end{array}$ & $\begin{array}{l}\text { rs1464510 } \\
\text { rs1738074 }\end{array}$ & $\begin{array}{l}0.521 \\
0.459\end{array}$ & $\begin{array}{l}0.500 \\
0.395\end{array}$ & 508 & 888 \\
\hline Van Heel et al., 2008 [10] & British & Illumina Нар300 & $\begin{array}{c}\text { LPP } \\
\text { TAGAP }\end{array}$ & $\begin{array}{l}\text { rs1464510 } \\
\text { rs1738074 }\end{array}$ & $\begin{array}{l}0.519 \\
0.472\end{array}$ & $\begin{array}{l}0.457 \\
0.422\end{array}$ & 778 & 1422 \\
\hline
\end{tabular}

RT-PCR: transcriptase PCR; MAF: Minor allele frequency; SNP: single nucleotide polymorphism; Minor allele in LPP rs1464510 is A, and minor allele in TAGAP rs1738074 is A. 


\subsection{Risk of Bias Assessment}

The results of bias assessment are presented in Table 2. Each study was compliant with HWE. All studies had a low risk of bias from population stratification, selective outcome reports, ascertainment of celiac disease and ascertainment of control. The risk of bias was highest in quality control for genotyping and confounding bias (both unclear in 1 study, 14.29\%).

Table 2. The risk of bias assessment.

\begin{tabular}{|c|c|c|c|c|c|c|c|}
\hline Author, Year (Ref.) & $\begin{array}{c}\text { Ascertainment } \\
\text { of Celiac } \\
\text { Disease }\end{array}$ & $\begin{array}{l}\text { Ascertainment } \\
\text { of Control }\end{array}$ & $\begin{array}{c}\text { Quality } \\
\text { Control for } \\
\text { Genotyping }\end{array}$ & $\begin{array}{l}\text { Population } \\
\text { Stratification }\end{array}$ & $\begin{array}{l}\text { Confounding } \\
\text { Bias }\end{array}$ & $\begin{array}{c}\text { Selective } \\
\text { Outcome } \\
\text { Report }\end{array}$ & HWE \\
\hline $\begin{array}{c}\text { Plaza-Izurieta et al., } \\
2011 \text { [7] }\end{array}$ & Yes & Yes & Yes & Yes & Yes & Yes & Yes \\
\hline $\begin{array}{c}\text { Sperandeo et al., } \\
2011[31]\end{array}$ & Yes & Yes & Yes & Yes & Yes & Yes & Yes \\
\hline $\begin{array}{c}\text { Dubois et al., } \\
2010[8]\end{array}$ & Yes & Yes & Yes & Yes & Yes & Yes & Yes \\
\hline $\begin{array}{l}\text { Coenen et al., } \\
2009 \text { [30] }\end{array}$ & Yes & Yes & Yes & Yes & Yes & Yes & Yes \\
\hline $\begin{array}{l}\text { Romanos et al., } \\
2008 \text { [33] }\end{array}$ & Yes & Yes & Unclear & Yes & Unclear & Yes & Yes \\
\hline Hunt et al., 2008 [9] & Yes & Yes & Yes & Yes & Yes & Yes & Yes \\
\hline $\begin{array}{l}\text { Van Heel et al., } \\
2008 \text { [10] }\end{array}$ & Yes & Yes & Yes & Yes & Yes & Yes & Yes \\
\hline
\end{tabular}

HWE: Hard-Weinberg Equilibrium.

\subsection{Association between the LPP rs1464510 Polymorphism and CD Risk}

The seven studies reported an association between LPP rs1464510 polymorphism and CD, with 14,936 cases and 24,788 controls (Table 3). The pooled OR (A vs. C) showed moderate heterogeneity $\left(p=0.106\right.$, and $\left.\mathrm{I}^{2}=29.52 \%\right)$ across the studies, with a pooled OR of 1.26 (95\% CI: $1.22,1.30$ ) (part A of Figure 2), suggesting that individuals carrying the risk A allele had a $26 \%$ higher risk of developing CD than those carrying the C allele. The PAR for risk allele A was $10.72 \%$. The sensitivity analysis suggested that, if we excluded the study by Coenen et al. [30], $\mathrm{I}^{2}$ was reduced from $29.52 \%$ to $11.64 \%$ and the pooled odds ratio was 1.27 (95\% CI: 1.23, 1.31) (Supplementary Materials Table S3). The Egger test $(p=0.100)$ and Begg and Mazumdar rank correlation $(p=0.284)$ suggested that no publication bias existed. Publication bias was also tested using a funnel plot (Supplementary Materials Figure S1). The classic fail-safe N value was $1032(Z=14.21 ; p=0.00)$, which suggested that 1032 unpublished negative studies would have to be included to convert the combined $p$ value to a non-significant value. 
Table 3. Genotype frequencies for $L P P$ rs1464510and genotype effects of studies included in the meta-analysis.

\begin{tabular}{|c|c|c|c|c|c|c|c|c|c|c|c|c|c|c|}
\hline \multirow{2}{*}{ Author (Ref.) } & \multirow{2}{*}{ Country } & \multicolumn{3}{|c|}{ Case Genotype } & \multicolumn{3}{|c|}{ Control Genotype } & \multicolumn{2}{|c|}{ A vs. $\mathrm{C}$} & \multicolumn{2}{|c|}{ AA vs. CC } & \multicolumn{2}{|c|}{ AC vs. CC } & \multirow{2}{*}{ HWE } \\
\hline & & AA & AC & $\mathrm{CC}$ & AA & $\mathrm{AC}$ & $\mathrm{CC}$ & OR & $95 \% \mathrm{CI}$ & OR & $95 \% \mathrm{CI}$ & OR & $95 \% \mathrm{CI}$ & \\
\hline Plaza-Izurieta et al. [7] & Spain & 222 & 541 & 331 & 95 & 263 & 182 & 1.133 & $0.978-1.313$ & 1.258 & $0.951-1.736$ & 1.131 & $0.896-1.428$ & 0.999 \\
\hline Sperandeo et al. [31] & Italy & 152 & 324 & 161 & 108 & 362 & 241 & 1.420 & $1.219-1.653$ & 2.107 & $1.534-2.893$ & 1.340 & $1.044-1.719$ & 0.141 \\
\hline \multirow[t]{12}{*}{ Dubois et al. [8] } & UK1 & 201 & 368 & 168 & 526 & 1285 & 785 & 1.336 & $1.190-1.500$ & 1.786 & $1.415-2.253$ & 1.338 & $1.092-1.639$ & 0.997 \\
\hline & UK2 & 508 & 922 & 419 & 991 & 2441 & 1504 & 1.357 & $1.258-1.463$ & 1.840 & $1.580-2.142$ & 1.356 & $1.188-1.547$ & 0.992 \\
\hline & Finland 1 & 234 & 310 & 103 & 547 & 907 & 375 & 1.249 & $1.098-1.420$ & 1.557 & $1.193-2.033$ & 1.244 & $0.966-1.603$ & 0.978 \\
\hline & The Netherlands & 226 & 400 & 177 & 206 & 423 & 217 & 1.160 & $1.012-1.330$ & 1.345 & $1.023-1.769$ & 1.159 & $0.911-1.475$ & 0.996 \\
\hline & Italy 1 & 133 & 248 & 116 & 121 & 271 & 151 & 1.196 & $1.007-1.421$ & 1.431 & $1.013-2.021$ & 1.191 & $0.885-1.603$ & 0.977 \\
\hline & USA & 254 & 486 & 233 & 117 & 276 & 162 & 1.228 & $1.060-1.424$ & 1.509 & $1.122-2.031$ & 1.224 & $0.954-1.571$ & 0.978 \\
\hline & Hungary & 274 & 480 & 211 & 241 & 532 & 294 & 1.259 & $1.113-1.424$ & 1.584 & $1.237-2.029$ & 1.257 & $1.013-1.560$ & 0.991 \\
\hline & Ireland & 150 & 298 & 149 & 286 & 718 & 452 & 1.262 & $1.102-1.444$ & 1.591 & $1.214-2.086$ & 1.259 & $1.001-1.583$ & 0.977 \\
\hline & Poland & 138 & 282 & 144 & 146 & 355 & 215 & 1.188 & $1.016-1.389$ & 1.411 & $1.031-1.932$ & 1.186 & $0.912-1.542$ & 0.980 \\
\hline & Spain & 117 & 274 & 159 & 70 & 209 & 154 & 1.271 & $1.062-1.522$ & 1.619 & $1.118-2.343$ & 1.270 & $0.954-1.689$ & 0.948 \\
\hline & Italy 2 & 247 & 505 & 258 & 134 & 388 & 282 & 1.420 & $1.244-1.621$ & 2.015 & $1.539-2.638$ & 1.423 & $1.148-1.763$ & 0.978 \\
\hline & Finland 2 & 94 & 124 & 41 & 184 & 325 & 144 & 1.340 & $1.089-1.648$ & 1.794 & $1.171-2.749$ & 1.340 & $0.895-2.007$ & 0.983 \\
\hline Coenen et al. [30] & The Netherlands & 223 & 396 & 176 & 438 & 841 & 404 & 1.081 & $0.959-1.218$ & 1.169 & $0.920-1.485$ & 1.081 & $0.873-1.338$ & 0.994 \\
\hline Romanos et al. [33] & Italy & 145 & 269 & 124 & 133 & 296 & 164 & 1.201 & $1.018-1.416$ & 1.442 & $1.035-2.008$ & 1.202 & $0.903-1.600$ & 0.980 \\
\hline \multirow[t]{3}{*}{ Hunt et al. [9] } & UK & 192 & 359 & 168 & 311 & 771 & 479 & 1.327 & $1.171-1.504$ & 1.760 & $1.369-2.264$ & 1.328 & $1.070-1.647$ & 0.981 \\
\hline & Ireland & 97 & 208 & 111 & 192 & 473 & 292 & 1.153 & $0.980-1.357$ & 1.329 & $0.958-1.844$ & 1.157 & $0.881-1.519$ & 0.986 \\
\hline & The Netherlands & 138 & 253 & 117 & 222 & 444 & 222 & 1.086 & $0.931-1.267$ & 1.179 & $0.866-1.606$ & 1.081 & $0.824-1.419$ & 1.000 \\
\hline Van Heel et al. [10] & UK & 210 & 388 & 180 & 297 & 706 & 419 & 1.283 & $1.134-1.452$ & 1.646 & $1.284-2.110$ & 1.279 & $1.033-1.585$ & 0.990 \\
\hline Overall odds ratio & - & - & - & - & - & - & - & 1.258 & $1.221-1.296$ & 1.583 & $1.490-1.681$ & 1.255 & $1.192-1.321$ & - \\
\hline
\end{tabular}


$\mathbf{A}$

Study name country

Plaza-Izurieta L 2011 Spain

Sperandeo MP 2011 Italy

Dubois PC 2010

Dubois PC 2010

Dubois PC 2010

Dubois PC 2010

Dubois PC 2010

Dubois PC 2010

Dubois PC 2010

Dubois PC 2010

Dubois PC 2010

Dubois PC 2010

Dubois PC 2010

Coenen M.J 2009

Romanos J 2008

Hunt K.A 2008

Hunt K.A 2008

Hunt K.A 2008

Van Heel D. A 2008
Statistics for each study

Odds Lower Upper

ratio limit limit Z-Value p-Value

$\begin{array}{lllll}1.133 & 0.978 & 1.313 & 1.665 & 0.096\end{array}$

$\begin{array}{lll}1.420 & 1.219 & 1.653\end{array}$

$\begin{array}{lll}1.357 & 1.258 & 1.463\end{array}$

1.35

0.001

\begin{tabular}{llllll}
\hline & 1.228 & 1.060 & 1.424 & 2.728 & 0.006
\end{tabular}

$\begin{array}{llllll}\text { Hungary } & 1.259 & 1.113 & 1.424 & 3.658 & 0.000\end{array}$

Ireland $\quad \begin{array}{llllll}1.262 & 1.102 & 1.444 & 3.374 & 0.001\end{array}$

$\begin{array}{llllll}\text { Poland } & 1.188 & 1.016 & 1.389 & 2.156 & 0.031\end{array}$

$\begin{array}{lllllll}\text { Spain } & 1.271 & 1.062 & 1.522 & 2.609 & 0.009\end{array}$

$\begin{array}{llllll}\text { Italy } 2 & 1.420 & 1.244 & 1.621 & 5.195 & 0.000\end{array}$

$\begin{array}{llllll}\text { Finland } 2 & 1.340 & 1.089 & 1.648 & 2.772 & 0.006\end{array}$

$\begin{array}{llllll}\text { Netherland1.081 } & 0.959 & 1.218 & 1.279 & 0.201\end{array}$

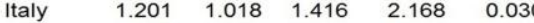

$\begin{array}{llllll}\text { UK } & 1.327 & 1.171 & 1.504 & 4.428 & 0.000\end{array}$

$\begin{array}{llllll}\text { Ireland } & 1.153 & 0.980 & 1.357 & 1.711 & 0.087\end{array}$

$\begin{array}{llllll}\text { Netherland1.086 } & 0.931 & 1.267 & 1.051 & 0.293\end{array}$

$\begin{array}{lllllllll} & \text { UK } & 1.283 & 1.934 & 1.452 & 3.944 & 0.000\end{array}$

$\begin{array}{rrrrr}1.283 & 1.134 & 1.452 & 3.944 & 0.000 \\ 1.258 & 1.221 & 1.296 & 15.042 & 0.000\end{array}$
Odds ratio and $95 \% \mathrm{Cl}$

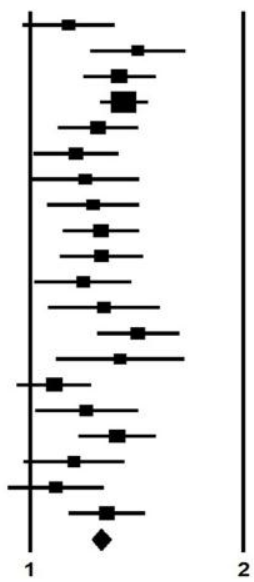

Odds ratio and $95 \% \mathrm{Cl}$

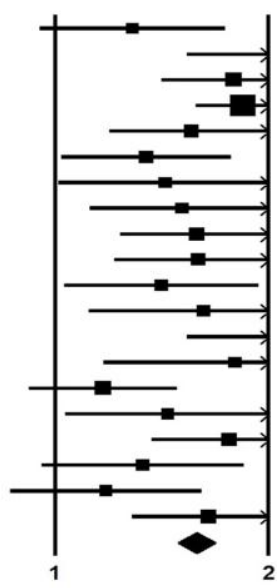

Odds ratio and $95 \% \mathrm{Cl}$

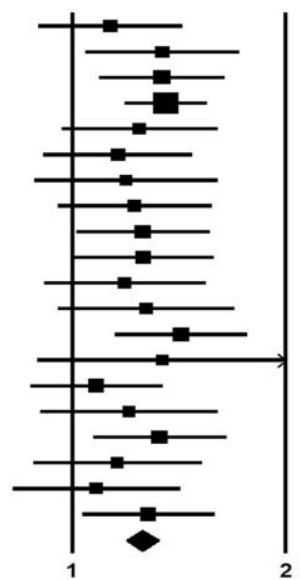

Figure 2. Forest plot of the association between LPP rs1464510 polymorphism and CD risk in (A) A vs. C; (B) AA vs.CC; (C) AC vs. CC. 
The genotype frequency and estimated ORs of LPP rs1464510 are presented in parts B and C of Figure 2. The $\mathrm{OR}_{1}$ (AA vs. CC) $\left(p=0.097 ; \mathrm{I}^{2}=30.45 \%\right)$ was moderately heterogeneous, and the $\mathrm{OR}_{2}$ (AC vs. CC) $\left(p=0.979 ; \mathrm{I}^{2}=0.0 \%\right)$ was homogenous. The pooled $\mathrm{OR}_{1}(1.58 ; 95 \%$ CI: $1.49,1.68$; $p<0.001)$ and $\mathrm{OR}_{2}(1.26 ; 95 \%$ CI: 1.19, 1.32; $p<0.001)$ were statistically significant, which indicated that persons with AA and AC genotypes in LPP rs1464510 had an approximately 58\% and $26 \%$ higher risk, respectively, of developing $C D$ than persons with the $C C$ genotype. The Egger test did not suggest any asymmetry for both ORs ( $p=0.133$ for $\mathrm{OR}_{1}, p=0.054$ for $\mathrm{OR}_{2}$ ). The $\lambda$ was 0.512 ( $95 \%$ CI: 0.388 , $0.660)$, suggesting that a co-dominant effect was most likely.

\subsection{Association between the TAGAP rs1738074 Polymorphism and CD Risk}

The seven studies reported an association between TAGAP rs1738074 polymorphism and CD, with 14,936 cases and 24,788 controls (Table 4). The pooled OR (A vs. G) was 1.17 (95\% CI: 1.14, 1.21 ), estimated by the fixed-effects model $\left(p=0.974\right.$, and $\left.\mathrm{I}^{2}=0.00 \%\right)$ (part A of Figure 3), which suggested that individuals carrying the risk A allele had a $17 \%$ higher risk of developing CD than those carrying the $\mathrm{G}$ allele. The PAR for risk allele A was 6.59\%. The Egger test $(p=0.440)$ and Begg and Mazumdar rank correlation $(p=0.315)$ suggested that no publication bias existed. Publication bias was also tested using a funnel plot (Supplementary Materials Figure S2). The classic fail-safe N value was $513(Z=10.11 ; p=0.00)$, which suggested that 513 unpublished negative studies would have to be included to convert the combined $p$ value to a non-significant value.

The $\mathrm{OR}_{1}$ (AA vs. GG, 1.37; 95\% CI: $1.29,1.46 ; p<0.001$ ) and the $\mathrm{OR}_{2}$ (AG vs. GG, $1.17 ; 95 \%$ CI: $1.11,1.22 ; p<0.001)$ were homogenous, and estimated by a fixed-effects model in parts $B$ and $C$ of Figure 3. The results can be interpreted as indicating that persons with AA and AG genotypes in TAGAP rs 1738074 had approximately $37 \%$ and $17 \%$ higher risks, respectively, of developing CD than persons with the GG genotype. Egger's test did not suggest any asymmetry for both ORs ( $p=0.425$ for $\mathrm{OR}_{1}, p=0.611$ for $\mathrm{OR}_{2}$ ). The $\lambda$ was 0.496 (95\% CI: 0.310, 0.711 ), which suggested that a co-dominant effect was most likely. 
Table 4. Genotype frequencies for TAGAP rs1738074 and genotype effects of studies included in the meta-analysis.

\begin{tabular}{|c|c|c|c|c|c|c|c|c|c|c|c|c|c|c|}
\hline \multirow{2}{*}{ Author (Ref.) } & \multirow{2}{*}{ Country } & \multicolumn{3}{|c|}{ Case Genotype } & \multicolumn{3}{|c|}{ Control Genotype } & \multicolumn{2}{|c|}{ A vs. $\mathrm{G}$} & \multicolumn{2}{|c|}{ AA vs. GG } & \multicolumn{2}{|c|}{ AG vs. GG } & \multirow{2}{*}{ HWE } \\
\hline & & AA & AG & GG & AA & AG & GG & OR & $95 \% \mathrm{CI}$ & OR & $95 \% \mathrm{CI}$ & OR & $95 \% \mathrm{CI}$ & \\
\hline Plaza-Izurieta et al. [7] & Spain & 196 & 534 & 364 & 89 & 261 & 190 & 1.071 & $0.924-1.242$ & 1.150 & $0.847-1.561$ & 1.068 & $0.849-1.343$ & 0.968 \\
\hline Sperandeo et al. [31] & Italy & 144 & 305 & 188 & 125 & 354 & 231 & 1.176 & $1.010-1.370$ & 1.415 & $1.041-1.925$ & 1.059 & $0.828-1.354$ & 0.596 \\
\hline \multirow[t]{12}{*}{ Dubois et al. [8] } & UK1 & 164 & 367 & 205 & 498 & 1278 & 820 & 1.145 & $1.019-1.286$ & 1.311 & $1.038-1.655$ & 1.149 & $0.948-1.392$ & 0.999 \\
\hline & UK2 & 417 & 922 & 510 & 947 & 2430 & 1559 & 1.160 & $1.075-1.252$ & 1.346 & $1.156-1.568$ & 1.160 & $1.023-1.315$ & 0.999 \\
\hline & Finland 1 & 120 & 317 & 210 & 324 & 892 & 613 & 1.039 & $0.914-1.182$ & 1.081 & $0.832-1.404$ & 1.037 & $0.847-1.270$ & 0.987 \\
\hline & The Netherlands & 159 & 397 & 247 & 132 & 404 & 310 & 1.230 & $1.071-1.413$ & 1.512 & $1.137-2.010$ & 1.233 & $0.993-1.532$ & 0.984 \\
\hline & Italy 1 & 107 & 247 & 143 & 93 & 263 & 187 & 1.227 & $1.032-1.460$ & 1.505 & $1.057-2.141$ & 1.228 & $0.930-1.623$ & 0.974 \\
\hline & USA & 215 & 485 & 273 & 99 & 271 & 185 & 1.213 & $1.045-1.407$ & 1.472 & $1.088-1.992$ & 1.213 & $0.955-1.540$ & 0.989 \\
\hline & Hungary & 166 & 469 & 330 & 148 & 498 & 421 & 1.197 & $1.055-1.358$ & 1.431 & $1.099-1.864$ & 1.201 & $0.992-1.455$ & 0.970 \\
\hline & Ireland & 149 & 299 & 149 & 311 & 724 & 421 & 1.163 & $1.017-1.331$ & 1.345 & $1.027-1.761$ & 1.167 & $0.927-1.469$ & 0.993 \\
\hline & Poland & 75 & 261 & 228 & 77 & 316 & 323 & 1.173 & $0.996-1.382$ & 1.380 & $0.962-1.978$ & 1.170 & $0.924-1.481$ & 0.982 \\
\hline & Spain & 108 & 271 & 171 & 69 & 208 & 156 & 1.194 & $0.997-1.430$ & 1.428 & $0.984-2.071$ & 1.189 & $0.896-1.576$ & 0.981 \\
\hline & Italy 2 & 215 & 502 & 293 & 145 & 393 & 266 & 1.160 & $1.017-1.324$ & 1.346 & $1.029-1.760$ & 1.160 & $0.938-1.434$ & 0.994 \\
\hline & Finland 2 & 52 & 128 & 79 & 116 & 318 & 219 & 1.115 & $0.908-1.369$ & 1.243 & $0.820-1.884$ & 1.116 & $0.803-1.551$ & 0.976 \\
\hline Coenen et al. [30] & The Netherlands & 154 & 392 & 249 & 269 & 808 & 606 & 1.180 & $1.046-1.332$ & 1.393 & $1.088-1.784$ & 1.181 & $0.976-1.429$ & 0.990 \\
\hline Romanos et al. [33] & Italy & 111 & 267 & 160 & 101 & 287 & 205 & 1.187 & $1.005-1.403$ & 1.408 & $1.003-1.978$ & 1.192 & $0.914-1.555$ & 0.974 \\
\hline \multirow[t]{3}{*}{ Hunt et al. [9] } & UK & 152 & 357 & 210 & 286 & 764 & 511 & 1.137 & $1.003-1.289$ & 1.293 & $1.003-1.667$ & 1.137 & $0.927-1.394$ & 0.988 \\
\hline & Ireland & 112 & 208 & 96 & 210 & 476 & 271 & 1.227 & $1.043-1.444$ & 1.506 & $1.086-2.087$ & 1.234 & $0.928-1.639$ & 0.971 \\
\hline & The Netherlands & 107 & 252 & 148 & 139 & 424 & 325 & 1.296 & $1.109-1.515$ & 1.679 & $1.222-2.308$ & 1.305 & $1.017-1.674$ & 0.971 \\
\hline Van Heel et al. [10] & UK & 173 & 388 & 217 & 253 & 694 & 475 & 1.223 & $1.080-1.385$ & 1.497 & $1.164-1.925$ & 1.224 & $0.999-1.499$ & 0.986 \\
\hline Overall odds ratio & - & - & - & - & - & - & - & 1.170 & $1.136-1.206$ & 1.370 & $1.289-1.457$ & 1.166 & $1.111-1.224$ & - \\
\hline
\end{tabular}


A

Study name country

Plaza-Izurieta L 2011 Spain Sperandeo MP 2011 Italy $\begin{array}{ll}\text { Sperandeo MP } 2011 & \text { Italy } \\ \text { Dubois PC 2010 } & \text { UK1 }\end{array}$ Dubois PC 2010 Dubois PC 2010
Dubois PC 2010 Dubois PC 2010
Dubois PC 2010 Dubois PC 2010 Dubois PC 2010 Dubois PC 2010 Dubois PC 2010 Dubois PC 2010 Dubois PC 2010 Dubois PC 2010 Dubois PC 2010 Dubois PC 2010 Coenen M.J 2009 Coenen M.J 2009
Romanos J 2008 Romanos J 200 Hunt K.A 2008 Hunt K.A 2008
Hunt K.A 2008 Van Heel D. A 2008

$\begin{array}{lrrrrr}\text { Italy } & 1.176 & 1.010 & 1.370 & 2.091 & 0.037 \\ \text { UK1 } & 1.145 & 1.019 & 1.286 & 2.285 & 0.022 \\ \text { UK2 } & 1.160 & 1.075 & 1.252 & 3.841 & 0.000 \\ \text { Finland 1 } & 1.039 & 0.914 & 1.182 & 0.591 & 0.554 \\ \text { Netherland } & 1.230 & 1.071 & 1.413 & 2.931 & 0.003 \\ \text { Italy 1 } & 1.227 & 1.032 & 1.460 & 2.311 & 0.021 \\ \text { USA } & 1.213 & 1.045 & 1.407 & 2.545 & 0.011 \\ \text { Hungary } & 1.197 & 1.055 & 1.358 & 2.799 & 0.005 \\ \text { Ireland } & 1.163 & 1.017 & 1.331 & 2.201 & 0.028 \\ \text { Poland } & 1.173 & 0.996 & 1.382 & 1.911 & 0.056 \\ \text { Spain } & 1.194 & 0.997 & 1.430 & 1.923 & 0.054 \\ \text { Italy 2 } & 1.160 & 1.017 & 1.324 & 2.205 & 0.027 \\ \text { Finland 2 } & 1.115 & 0.908 & 1.369 & 1.040 & 0.298 \\ \text { Netherland1.180 } & 1.046 & 1.332 & 2.693 & 0.007 \\ \text { Italy } & 1.187 & 1.005 & 1.403 & 2.020 & 0.043 \\ \text { UK } & 1.137 & 1.003 & 1.289 & 2.007 & 0.045 \\ \text { Ireland } & 1.227 & 1.043 & 1.444 & 2.461 & 0.014 \\ \text { Netherland1.296 } & 1.109 & 1.515 & 3.264 & 0.001 \\ \text { UK } & 1.223 & 1.080 & 1.385 & 3.179 & 0.001 \\ & 1.170 & 1.136 & 1.206 & 10.252 & 0.000\end{array}$

B
Study name country

Plaza-Izurieta L 2011 Spain Sperandeo MP 2011 Italy Dubois PC 2010

Dubois PC 2010

Dubois PC 2010

Dubois PC 2010

Dubois PC 2010

Dubois PC 2010

Dubois PC 2010

Dubois PC 2010

Dubois PC 2010

Dubois PC 2010

Dubois PC 2010

Coenen M.J 2009

Romanos J 2008

Hunt K.A 2008

Hunt K.A 2008

Hunt K.A 2008

Van Heel D. A 2008
Dubois PC 2010

UK1

$\begin{array}{llll}\text { UK2 } & 1.346 & 1.156 & 1.568 \\ \text { Finland 1 } & 1.081 & 0.832 & 1.404 \\ \text { Netherland } 1.512 & 1.137 & 2.010\end{array}$

$\begin{array}{llll}\text { Italy } 1 & 1.505 & 1.057 & 2.141\end{array}$

$\begin{array}{llll}\text { USA } & 1.472 & 1.088 & 1.992 \\ \text { Hungary } & 1.431 & 1.099 & 1.864\end{array}$

$\begin{array}{lllll}\text { Hungary } & 1.431 & 1.099 & 1.864 \\ \text { Ireland } & 1.345 & 1.027 & 1.761\end{array}$

\begin{tabular}{llll} 
Poland & 1.380 & 0.962 & 1.978 \\
\hline
\end{tabular}

$\begin{array}{lllll}\text { Spain } & 1.428 & 0.984 & 2.071\end{array}$

\begin{tabular}{llll} 
Italy 2 & 1.346 & 1.029 & 1.760 \\
\hline
\end{tabular}

\begin{tabular}{llll} 
Finland 2 & 1.243 & 0.820 & 1.884 \\
\hline
\end{tabular}

$\begin{array}{lrrr}\text { Netherland1.393 } & 1.088 & 1.784 \\ \text { Italy } 1.408 & 1.003 & 1.978\end{array}$

$\begin{array}{llll}\text { Italy } & 1.408 & 1.003 & 1.978 \\ \text { UK } & 1.293 & 1.003 & 1.667\end{array}$

$\begin{array}{llll}\text { Ireland } & 1.506 & 1.086 & 2.087\end{array}$

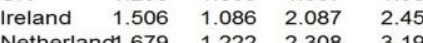

\begin{tabular}{lllll} 
Netherland1.679 & 1.222 & 2.308 & 3.194 \\
\hline$K$ & 1.497 & 1.164 & 1.925 & 3.145
\end{tabular}

$\begin{array}{rrrr}1.497 & 1.164 & 1.925 & 3.145 \\ 1.370 & 1.289 & 1.457 & 10.080\end{array}$
Odds ratio and $95 \% \mathrm{Cl}$

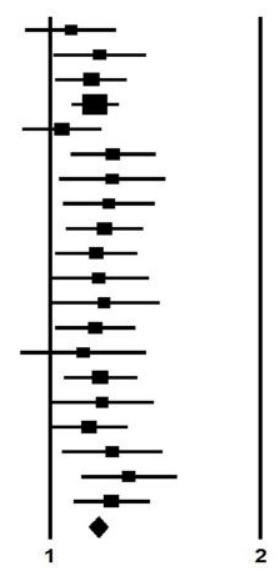

Odds ratio and $95 \% \mathrm{Cl}$

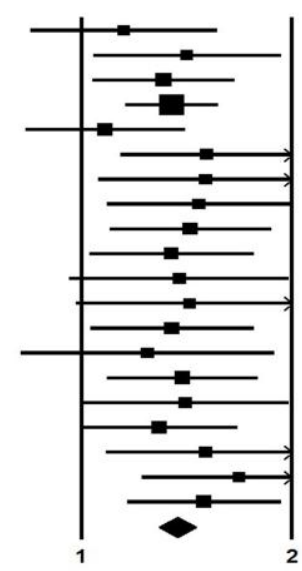

Odds ratio and $95 \% \mathrm{Cl}$

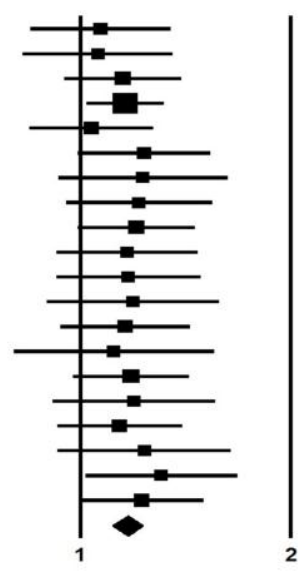

Figure 3. Forest plot of the association between TAGAP rs1738074 polymorphism and CD risk in (A) A vs. G; (B) AA vs.GG; (C) AG vs. GG. 


\section{Discussion}

Our meta-analysis suggests that both LPP rs1464510 and TAGAP rs1738074 polymorphisms contribute to the susceptibility to $\mathrm{CD}$ in European and American.

The pooled OR (A vs. C) of LPP suffered from moderate heterogeneity, but $\mathrm{I}^{2}$ decreased significantly (from $29.52 \%$ to $11.63 \%$ ) when we eliminated The Netherlands data from Coenen et al. [30], indicating that heterogeneity originated mainly from this study. The results between different studies are often heterogeneous, and there are three feasible reasons for such heterogeneity in genetic association studies: association in one population rather than in another, different studies without comparable measures of phenotype, or deviation from HWE [34]. Therefore, we speculate that the main underlying cause of heterogeneity might be populations of various ethnicities.

$L P P$, which is strongly expressed in the small intestine, participates in the regulation of cell adhesion, cytoskeletal remodeling and maintenance of cell shape and motility [35,36], and it seems to be activated more strongly in biopsy specimens from $C D$ patients than in those from non-CD controls [7]. We infer that mutations in the LPP lead to the PTP1B becoming sufficient or even excessive, so more ERK may be activated, and that it may play a functional role in $C D$ enterocyte proliferation. Our results suggested a powerful relationship between CD and the LPP of rs1464510 $(p<0.001$, $\mathrm{OR}=1.26,95 \% \mathrm{CI}: 1.22-1.30)$.TAGAP is a Rho GTPase-activating protein crucial for modulating cytoskeletal changes $[9,11,12]$, and it is thought to be a negative regulator of cell signaling and relevant to the regulation of the Rho GTPase cycle [37]. Therefore, we hypothesize that mutations in the TAGAP rs1738074 might increase GTPase activity, which propagates the inactive form of the Rho molecule in the Rho GTPase cycle and leads to negative regulation of downstream effects, thus promoting the development of CD. Our meta-analysis confirmed the involvement of rs 1738074 in CD susceptibility $(p<0.001, \mathrm{OR}=1.17,95 \% \mathrm{CI}: 1.14-1.21)$, so pathway analysis should be implemented to generate hypotheses for clarifying the biological link between TAGAP and CD [38].

There are some limitations of our study. First, we only included European (38197/39725) and American (1528/39725) populations; nonetheless, our results provide a comprehensive overview of the association between LPP rs1464510/TAGAP rs1738074 and CD in European populations. Second, all included studies were case-control studies, which might have overestimated the genetic association; a population-based nested case-control study is needed to avoid this bias. Finally, because only English-language literature was retrieved, we may have missed relevant articles written in other languages.

\section{Conclusions}

In summary, our meta-analysis reveals that both LPP rs1464510 and TAGAP rs1738074 are associated with $C D$ susceptibility. Furthermore, the gene-gene and gene-environment interactions should be evaluated, and studies with larger and more diverse samples should be performed to confirm the results of this meta-analysis.

Supplementary Materials: The following are available online at www.mdpi.com/1660-4601/14/2/171/s1, Figure S1: Funnel plot for LPP rs1464510 (A vs. C) with CD. Figure S2: Funnel plot for TAGAP rs1738074 (A vs. G) with CD. Table S1: Risk of bias assessment for genetic association studies of CD of studies included in the meta-analysis. Table S2: MOOSE checklist: The association of LPP and TAGAP genes with CD risks: a meta-analysis. Table S3: The sensitivity analysis of $L P P$ rs1464510 and CD risk (A vs. C).

Acknowledgments: This work was supported in part by Training Program of the Major Research Plan of the National Natural Science Foundation of China (Grant numbers: 91543132), National Natural Science Foundation of China (Grant numbers: 30901249, 81101267 and 81541070), Guangdong Natural Science Foundation (Grant numbers: 10151063201000036,S2011010002526and 2016A030313089), Guangdong Province Medical Research Foundation (Grant number: A2014374 and A2015310) and Project from Jinan university (Grant number: 21612426, 21615426, JNUPHPM2016001, JNUPHPM2016002). 
Author Contributions: Shi-Qi Huang and Na Zhang contributed equally to writing of this paper. Chun-Xia Jing, Guang Yang and Eddy Y. Zeng contributed to study conception and design. Zi-Xing Zhou, Chui-Can Huang, Cheng-Li Zeng, Di Xiao, Cong-Cong Guo, Ya-Jing Han, Xiao-Hong Ye, Xing-Guang Ye, Mei-Ling Ou, Bao-Huan Zhang, Yang Liu performed the part of analysis and the interpretation. All authors approved the final version to be published.

Conflicts of Interest: The authors declare no conflict of interest.

\section{References}

1. Ludvigsson, J.F.; Leffler, D.A.; Bai, J.C.; Biagi, F.; Fasano, A.; Green, P.H.; Hadjivassiliou, M.; Kaukinen, K.; Kelly, C.P.; Leonard, J.N.; et al. The Oslo definitions for coeliac disease and related terms. Gut 2013, 62, 43-52. [CrossRef] [PubMed]

2. Catassi, C.; Gatti, S.; Fasano, A. The new epidemiology of celiac disease. J. Pediatr. Gastroenterol. Nutr. 2014, 59, S7-S9. [CrossRef] [PubMed]

3. Van De Kamer, J.H.; Weijers, H.A. Coeliac disease. V. Some experiments on the cause of the harmful effect of wheat gliadin. Acta Paediatr. 1955, 44, 465-469. [CrossRef] [PubMed]

4. Sollid, L.M. Coeliac disease: Dissecting a complex inflammatory disorder. Nat. Rev. Immunol. 2002, 2, 647-655. [CrossRef] [PubMed]

5. Castellanos-Rubio, A.; Martin-Pagola, A.; Santín, I.; Hualde, I.; Aransay, A.M.; Castaño, L.; Vitoria, J.C.; Bilbao, J.R. Combined functional and positional gene information for the identification of susceptibility variants in celiac disease. Gastroenterology 2008, 134, 738-746. [CrossRef] [PubMed]

6. Van Heel, D.A.; Hunt, K.; Greco, L.; Wijmenga, C. Genetics in coeliac disease. Best Pract. Res. Clin. Gastroenterol. 2005, 19, 323-339. [CrossRef] [PubMed]

7. Plaza-Izurieta, L.; Castellanos-Rubio, A.; Irastorza, I.; Fernandez-Jimenez, N.; Gutierrez, G.; Bilbao, J.R. Revisiting genome wide association studies (GWAS) in coeliac disease: Replication study in spanish population and expression analysis of candidate genes. J. Med. Genet. 2011, 48, 493-496. [CrossRef] [PubMed]

8. Dubois, P.C.A.; Trynka, G.; Franke, L.; Hunt, K.A.; Romanos, J.; Curtotti, A.; Zhernakova, A.; Heap, G.A.R.; Ádány, R.; Aromaa, A.; et al. Multiple common variants for celiac disease influencing immune gene expression. Nat. Genet. 2010, 42, 295-302. [CrossRef] [PubMed]

9. Hunt, K.A.; Zhernakova, A.; Turner, G.; Heap, G.A.; Franke, L.; Bruinenberg, M.; Romanos, J.; Dinesen, L.C.; Ryan, A.W.; Panesar, D.; et al. Newly identified genetic risk variants for celiac disease related to the immune response. Nat. Genet. 2008, 40, 395-402. [CrossRef] [PubMed]

10. Van Heel, D.A.; Franke, L.; Hunt, K.A.; Gwilliam, R.; Zhernakova, A.; Inouye, M.; Wapenaar, M.C.; Barnardo, M.C.N.M.; Bethel, G.; Holmes, G.K.T.; et al. A genome-wide association study for celiac disease identifies risk variants in the region harboring IL2 and IL21. Nat. Genet. 2007, 39, 827-829. [CrossRef] [PubMed]

11. Chen, R.; Stahl, E.A.; Kurreeman, F.A.S.; Gregersen, P.K.; Siminovitch, K.A.; Worthington, J.; Padyukov, L.; Raychaudhuri, S.; Plenge, R.M. Fine mapping the TAGAP risk locus in rheumatoid arthritis. Genes Immun. 2011, 12, 314-318. [CrossRef] [PubMed]

12. Festen, E.A.; Goyette, P.; Green, T.; Boucher, G.; Beauchamp, C.; Trynka, G.; Dubois, P.C.; Lagace, C.; Stokkers, P.C.; Hommes, D.W.; et al. A meta-analysis of genome-wide association scans identifies IL18RAP, PTPN2, TAGAP, and PUS10 as shared risk loci for Crohn's disease and celiac disease. PLoS Genet. 2011, 7, e1001283. [CrossRef] [PubMed]

13. Smyth, D.J.; Plagnol, V.; Walker, N.M.; Cooper, J.D.; Downes, K.; Yang, J.H.M.; Howson, J.M.M.; Stevens, H.; McManus, R.; Wijmenga, C.; et al. Shared and distinct genetic variants in type 1 diabetes and celiac disease. N. Engl. J. Med. 2008, 359, 2767-2777. [CrossRef] [PubMed]

14. Eyre, S.; Hinks, A.; Bowes, J.; Flynn, E.; Martin, P.; Wilson, A.G.; Morgan, A.W.; Emery, P.; Steer, S.; Hocking, L.J.; et al. Overlapping genetic susceptibility variants between three autoimmune disorders: Rheumatoid arthritis, type 1 diabetes and coeliac disease. Arthritis Res. Ther. 2010. [CrossRef] [PubMed]

15. Hinks, A.; Martin, P.; Flynn, E.; Eyre, S.; Packham, J.; Barton, A.; Worthington, J.; Thomson, W. Investigation of type 1 diabetes and coeliac disease susceptibility loci for association with juvenile idiopathic arthritis. Ann. Rheum. Dis. 2010, 69, 2169-2172. [CrossRef] [PubMed] 
16. Hue, S.; Mention, J.J.; Monteiro, R.C.; Zhang, S.; Cellier, C.; Schmitz, J.; Verkarre, V.; Fodil, N.; Bahram, S.; Cerf-Bensussan, N.; et al. A direct role for NKG2D/MICA interaction in villous atrophy during celiac disease. Immunity 2004, 21, 367-377. [CrossRef] [PubMed]

17. Holmgren Peterson, K.; Magnusson, K.E.; Stenhammar, L.; Falth-Magnusson, K. Confocal laser scanning microscopy of small-intestinal mucosa in celiac disease. Scand. J. Gastroenterol. 1995, 30, 228-234. [CrossRef] [PubMed]

18. Goldmann, W.H. Mechanical aspects of cell shape regulation and signaling. Cell Biol. Int. 2002, 26, 313-317. [CrossRef] [PubMed]

19. Bhattacharya, S.; Nanayakkara, M.; Kosova, R.; Lania, G.; Sarno, M.; Gaito, A.; Galatola, M.; Greco, L.; Cuomo, M.; Troncone, R.; et al. A celiac cellular phenotype, with altered LPP sub-cellular distribution, is inducible in controls by the toxic gliadin peptide p31-43. PLOS ONE 2013, 8, e79763.

20. Mertins, P.; Eberl, H.C.; Renkawitz, J.; Olsen, J.V.; Tremblay, M.L.; Mann, M.; Ullrich, A.; Daub, H. Investigation of protein-tyrosine phosphatase $1 \mathrm{~B}$ function by quantitative proteomics. Mol. Cell. Proteom. 2008, 7, 1763-1777. [CrossRef] [PubMed]

21. Dube, N.; Cheng, A.; Tremblay, M.L. The role of protein tyrosine phosphatase 1B in ras signaling. Proc. Natl. Acad. Sci. USA. 2004, 101, 1834-1839. [CrossRef] [PubMed]

22. Trackman, P.C.; Nanayakkara, M.; Lania, G.; Maglio, M.; Kosova, R.; Sarno, M.; Gaito, A.; Discepolo, V.; Troncone, R.; Auricchio, S.; et al. Enterocyte proliferation and signaling are constitutively altered in celiac disease. PLOS ONE 2013, 8, e76006.

23. Connelly, T.M.; Berg, A.S.; Harris, L.R.; Hegarty, J.P.; Ruggiero, F.M.; Deiling, S.M.; Brinton, D.L.; Koltun, W.A. T-cell activation Rho GTPase-activating protein expression varies with inflammation location and severity in Crohn's disease. J. Surg. Res. 2014, 190, 457-464. [CrossRef] [PubMed]

24. Connelly, T.M.; Sehgal, R.; Berg, A.S.; Hegarty, J.P.; Deiling, S.; Stewart, D.B.; Poritz, L.S.; Koltun, W.A. Mutation in tagap is protective of anal sepsis in ileocolic crohn's disease. Dis. Colon Rectum 2012, 55, 1145-1152. [CrossRef] [PubMed]

25. Moon, S.Y.; Zheng, Y. Rho GTPase-activating proteins in cell regulation. Trends Cell Biol. 2003, 13, 13-22. [CrossRef]

26. Zheng, Y. Dbl family guanine nucleotide exchange factors. Trends Biochem. Sci. 2001, 26, 724-732. [CrossRef]

27. Lamarche, N.; Hall, A. Gaps for rho-related GTPases. Trends Genet. 1994, 10, 436-440. [CrossRef]

28. Mardilovich, K.; Olson, M.F.; Baugh, M. Targeting rho GTPase signaling for cancer therapy. Future Oncol. 2012, 8, 165-177. [CrossRef] [PubMed]

29. Ligeti, E.; Welti, S.; Scheffzek, K. Inhibition and termination of physiological responses by gtpase activating proteins. Physiol. Rev. 2012, 92, 237-272. [CrossRef] [PubMed]

30. Coenen, M.J.H.; Trynka, G.; Heskamp, S.; Franke, B.; van Diemen, C.C.; Smolonska, J.; van Leeuwen, M.; Brouwer, E.; Boezen, M.H.; Postma, D.S.; et al. Common and different genetic background for rheumatoid arthritis and coeliac disease. Hum. Mol. Genet. 2009, 18, 4195-4203. [CrossRef] [PubMed]

31. Sperandeo, M.P.; Tosco, A.; Izzo, V.; Tucci, F.; Troncone, R.; Auricchio, R.; Romanos, J.; Trynka, G.; Auricchio, S.; Jabri, B.; et al. Potential celiac patients: A model of celiac disease pathogenesis. PLoS ONE 2011, 6, e21281. [CrossRef] [PubMed]

32. Thakkinstian, A.; McKay, G.J.; McEvoy, M.; Chakravarthy, U.; Chakrabarti, S.; Silvestri, G.; Kaur, I.; Li, X.; Attia, J. Systematic review and meta-analysis of the association between complement component 3 and age-related macular degeneration: A huge review and meta-analysis. Am. J. Epidemiol. 2011, 173, 1365-1379. [CrossRef] [PubMed]

33. Romanos, J.; Barisani, D.; Trynka, G.; Zhernakova, A.; Bardella, M.T.; Wijmenga, C. Six new coeliac disease loci replicated in an italian population confirm association with coeliac disease. J. Med. Genet. 2008, 46, 60-63. [CrossRef] [PubMed]

34. Munafò, M.R.; Flint, J. Meta-analysis of genetic association studies. Trends Genet. 2004, 20, 439-444. [CrossRef] [PubMed]

35. Petit, M.M.R. The focal adhesion and nuclear targeting capacity of the LIM-containing lipoma-preferred partner (LPP) protein. J. Biol. Chem. 2002, 278, 2157-2168. [CrossRef] [PubMed]

36. Jin, L.; Kern, M.J.; Otey, C.A.; Wamhoff, B.R.; Somlyo, A.V. Angiotensin II, focal adhesion kinase, and PRX1 enhance smooth muscle expression of lipoma preferred partner and its newly identified binding partner palladin to promote cell migration. Circ. Res. 2007, 100, 817-825. [CrossRef] [PubMed] 
37. Feighery, C.F.; McManus, R. Session 3: Joint nutrition society and irish nutrition and dietetic institute symposium on "nutrition and autoimmune disease". Proc. Nutr. Soc. 2009, 68, 122. [CrossRef] [PubMed]

38. Kumar, V.; Wijmenga, C.; Withoff, S. From genome-wide association studies to disease mechanisms: Celiac disease as a model for autoimmune diseases. Semin. Immunopathol. 2012, 34, 567-580. [CrossRef] [PubMed]

(c) 2017 by the authors; licensee MDPI, Basel, Switzerland. This article is an open access article distributed under the terms and conditions of the Creative Commons Attribution (CC BY) license (http:/ / creativecommons.org/licenses/by/4.0/). 